\title{
PET is prognostic of survival in pancreatic cancer patients
}

Patients with pancreatic cancer have a dismal prognosis and no curative treatment exists for those with unresectable disease. Currently, there are no pretreatment characteristics that predict disease outcomes. Researchers have now shown that preradiation PET scan parameters are prognostic of outcome. "We found that PET parameters were highly prognostic for patients with locally advanced unresectable pancreas cancer" explains Daniel Chang, senior investigator of the study.

In this study, 55 patients were treated with stereotactic body radiotherapy, and prior to treatment a PET scan was performed and parameters such as maximum selective uptake value $\left(\mathrm{SUV}_{\text {max }}\right)$ and total metabolic tumor burden (MTB) were analyzed. The median survival for patients with a low $\mathrm{SUV}_{\text {max }}$ value was 15.3 months and survival for those with a high SUV ${ }_{\text {max }}$ value was 9.8 months $(P<0.01)$. In the low MTB and the high
MTB groups the median survival rates were 18.0 and 10.1 months $(P<0.01)$. When patients were subgrouped according to their SUV ${ }_{\text {max }}$ values, patients with values $<5$ had longer progression-free and overall survival than those with higher values.

"The results are compelling in that they give us pretreatment knowledge on prognosis. For patients who have a poor prognosis, a strategy mainly focused on systemic chemotherapy makes sense. For patients with a better prognosis, local disease control becomes an important goal of therapy, and using radiation for these patients is appropriate," concludes Chang.

\section{Lisa Hutchinson}

Original article Schellenberg, D. et al. ${ }^{18}$ Fluorodeoxyglucose PET is prognostic of progression-free and overall survival in locally advanced pancreas cancer treated with stereotactic radiotherapy. Int. J. Radiat. Oncol. Biol. Phys. 77,

1420-1425 (2010) 